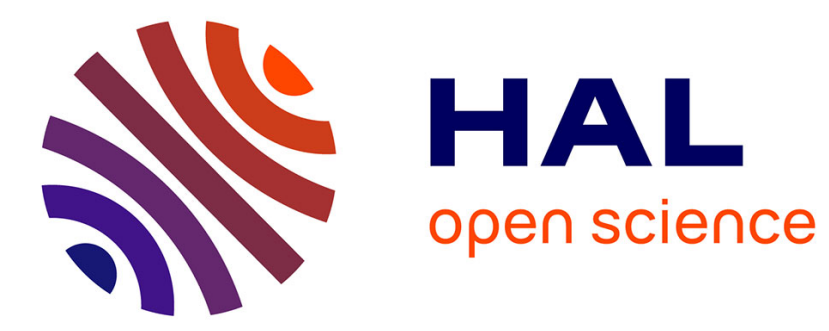

\title{
Passive yet Expressive TouchTokens
}

Rafael Morales González, Caroline Appert, Gilles Bailly, Emmanuel Pietriga

\section{To cite this version:}

Rafael Morales González, Caroline Appert, Gilles Bailly, Emmanuel Pietriga. Passive yet Expressive TouchTokens. Proceedings of the 35th SIGCHI conference on Human Factors in computing systems, May 2017, Denver, United States. pp.3741 - 3745, 10.1145/3025453.3025894 . hal-01562021

\section{HAL Id: hal-01562021 \\ https://hal.inria.fr/hal-01562021}

Submitted on 13 Jul 2017

HAL is a multi-disciplinary open access archive for the deposit and dissemination of scientific research documents, whether they are published or not. The documents may come from teaching and research institutions in France or abroad, or from public or private research centers.
L'archive ouverte pluridisciplinaire $\mathbf{H A L}$, est destinée au dépôt et à la diffusion de documents scientifiques de niveau recherche, publiés ou non, émanant des établissements d'enseignement et de recherche français ou étrangers, des laboratoires publics ou privés. 


\title{
Passive yet Expressive TouchTokens
}

\author{
Rafael Morales González ${ }^{1}$ \\ ${ }^{1}$ LRI, Univ. Paris-Sud, CNRS, \\ INRIA, \\ Caroline Appert ${ }^{1} \quad$ Gilles Bailly ${ }^{2,3}$ \\ ${ }^{2}$ LTCI, CNRS, \\ Telecom ParisTech, \\ Université Paris-Saclay, Orsay, France Université Paris-Saclay, Paris, France \\ Emmanuel Pietriga ${ }^{1}$ \\ ${ }^{3}$ Sorbonne Universités \\ UPMC Univ Paris 06, \\ CNRS, ISIR, Paris, France
}

\begin{abstract}
TouchTokens are passive tokens that can be recognized on any capacitive surface based on the spatial configuration of the fingers that hold them. However, interaction with these tokens is confined to the basic two-state model of touch interaction as the system only knows the tokens' position and cannot detect tokens that are not touched. We increase the expressive power of TouchTokens by introducing laser-cut lattice hinges in their design, so as to make them flexible. A new recognizer, that analyzes the micro-movements of the fingers that hold the tokens, enables the system to detect when a token is left on the surface rather than taken off it. It can also detect bend events that can be mapped to command triggers, and a squeezed state that can be used for quasi-modal interaction.
\end{abstract}

\section{ACM Classification Keywords}

H.5.2 : User Interfaces - Input devices and strategies.

\section{Author Keywords}

Tangible interaction; Multi-Touch input; Micro-movements

\section{INTRODUCTION}

TouchTokens [9] provide a simple means to develop tangible interfaces. The approach relies on easy-to-make passive tokens that feature notches constraining how users grasp them. Manipulating the tokens while maintaining the fingers in contact with the touch-sensitive surface leads to specific multitouch spatial patterns that can be uniquely identified using a relatively simple software recognizer. However, users are limited in how they can manipulate these tokens, as is often the case with approaches based on capacitive sensing.

In this article, we aim at increasing the expressive power of TouchTokens by making the system able to detect: 1) when a token is left on or lifted off the surface, 2) when it is squeezed and 3) when it is bent. We achieve this without introducing any kind of instrumentation, thus preserving the simplicity of the original approach, which relies exclusively on passive tokens, and which works with any off-the-shelf capacitive surface. Our solution relies on the hardware side on making

Rafael Morales González, Caroline Appert, Gilles Bailly \& Emmanuel Pietriga. Passive yet Expressive TouchTokens. In CHI '17: Proceedings of the 35th Annual ACM Conference on Human Factors in Computing Systems, 3741-3745, ACM, May 2017.

(C)ACM, 2017. This is the authors version of the work. It is posted here by permission of ACM for your personal use. Not for redistribution. The definitive version will be published in CHI '17, Denver, CO, USA.

http://dx. doi . org/10.1145/3025453.3025894 the tokens flexible by introducing lattice-hinges in their design, and on the software side on a novel recognizer that analyzes the micro-movements of the token-holding fingers that remain in contact with the surface.

After a short overview of related work, we describe the design of our flexible tokens, based on lattice hinges which can easily be obtained using fabrication processes such as laser cutting. We then report on a formative study in which we collected a sample of finger micro-movements that are representative of the manipulations afforded by our flexible tokens. Finally, we describe our recognizer, and evaluate its performance.

\section{RELATED WORK}

The most common approach to enabling tangible interaction on surfaces that use diffuse illumination technology consists in augmenting the objects with fiducial markers, and using a vision-based algorithm to identify them and track their location (see, e.g., [5]). Other projects have investigated tangibles that reflect incoming light to the surface in a specific way in order to support more manipulations, such as TZee tangibles [14], which have the shape of a truncated pyramid and support gesturing on their sides, or Lumino blocks [1], which can be stacked. Diffuse illumination is a solution that is usually reserved to large setups such as tabletops.

Another approach involves augmenting tangibles with magnets. When coupled with a force-resistive screen, the system can detect pressure and gestures performed on top of the tokens [6]. When coupled with a surface augmented with a Hall sensor grid, the system can track tokens hovering over the surface [8]. GaussBricks [7], which also rely on a display equipped with Hall sensors, are bricks that can be assembled together to create larger objects featuring both deformable and rigid parts. While this approach enables very rich interactions, it requires augmenting the surface with specific sensors, and ensuring that the device's environment is free of any ferrous object that could interfere with the tangibles' magnetic field.

Solutions based on capacitive sensing are more affordable, but usually more limited. The system will often only be able to track the tokens that users are touching. There are, however, a few exceptions that go beyond these limitations. CapStones and ZebraWidgets [3] are capacitive units that can be assembled to configure different conductive circuits, enabling more manipulations with the tangibles that can, for example, be stacked or feature moving parts. PUCs [13] widgets rely on the principle of mutual capacitance so as to be detected 
(a)

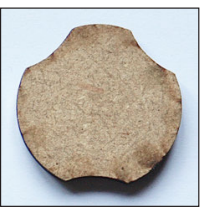

(b)

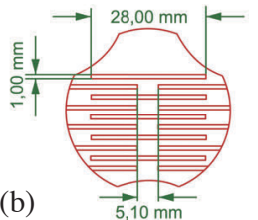

(c)

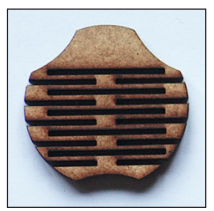

Figure 1. Making a TouchToken flexible: (a) original, rigid TouchToken (circle, $4 \mathrm{~cm}$ in diameter), (b) schematics of lattice-hinges, (c) flexible TouchToken. Vector descriptions of all flexible TouchTokens available at https: //www . lri . fr/ appert/touchtokens/index . html.

even when users do not touch them. However, after a moment, PUCs get rejected by the adaptive filtering method of capacitive screens. To avoid this issue, PERCs [12] are equipped with sensors to capture the electrical field emitted by the capacitive screen, enabling them to know if they are on the surface or not, and communicate their state (on vs. off the surface) to the system via the Bluetooth protocol. Our contribution also aims at increasing the number of possible interactions with tokens but, as described in the next section, we do so without relying on any advanced design or embedded electronics.

\section{MAKING TOUCHTOKENS MORE EXPRESSIVE}

We contribute three novel primitives to the interaction vocabulary of TouchTokens: a state (on/off), a quasi-mode (squeezed) and a discrete event (bent). We achieve this with a novel design that makes the tokens flexible, and with an analysis of the micro-movements users make when performing these interactions, following an approach similar to the recognizers designed to detect thumb-tip micro-gestures [2, 10]. This section describes our new tokens and introduces our hypotheses regarding the micro-movements we expect to observe.

\section{Designing Flexible TouchTokens}

Figure 1 shows our novel set of tokens, which can be squeezed or bent by pinching them. Laser-cutting lattice hinges is a common method in the maker community to make a piece of wood flexible using laser cutting. In our case, we performed several design iterations so as to make the tokens comfortable to manipulate while ensuring enough robustness. The kerfs' orientation was chosen so as to match that of the comfortable pinch formed by the thumb on one side and the \{index, middle $\}$ couple of fingers on the other side. The kerfs' width, length and interspacing provide enough elasticity to make the tokens easy to deform without requiring too high a force, while ensuring that they revert to their original shape when not pinched. We also considered resistance to avoid accidental pinches during regular manipulations, and robustness to avoid the risk of breaking.

\section{Detecting Tokens' on/off State}

Making the system aware of whether a token is still on the surface, or if it has been lifted off it, is an important feature of tangible interaction. It allows users to lay out several tokens on the surface (as in, e.g., Facet-streams [4]). Conductive tokens usually rely on the fact that the human body is a conductor. They thus become invisible to the system as soon as

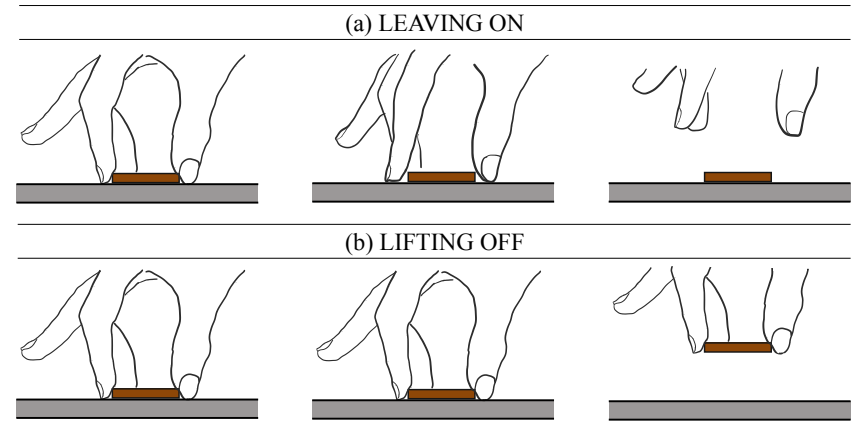

Figure 2. Finger micro-movements when leaving a token on the surface (a), and when lifting it off (b).

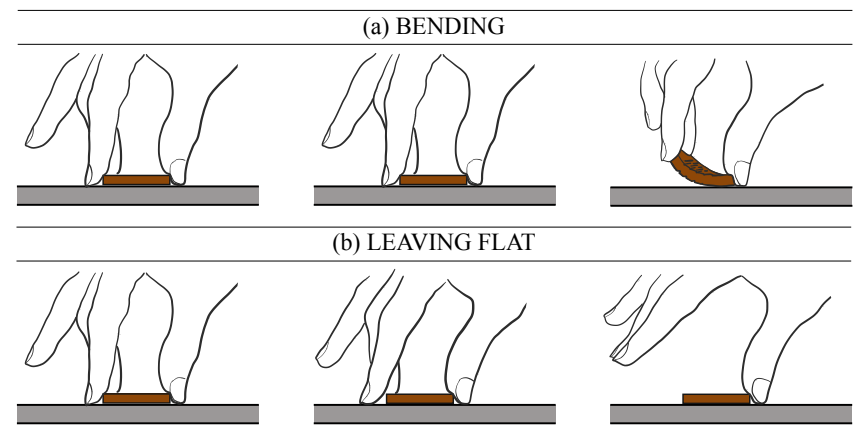

Figure 3. Micro-movements when (a) bending a token, (b) leaving it flat.

users no longer touch them. The system does not even know whether a token has been left on the surface or removed off it.

TouchTokens require users to both hold them by putting their fingers in the notches and touch the surface with those fingers. We hypothesized that the micro-movements made by the fingers at the time they leave the surface would have a distinct signature, depending on whether users were leaving tokens on the surface or were lifting them off. Figure 2 illustrates our hypothesis: when leaving a token on the surface, users are likely going to relax their grasp, while when lifting it off, they will likely maintain a firm grip, potentially compressing the token a bit. In the former case, we should observe finger traces that move slightly away from the touch points' centroid. In the latter case, we should observe finger traces that either remain still or move slightly toward the touch points' centroid.

\section{Squeezing Tokens}

When squeezing a token, the user's fingers remain in contact with the surface throughout the corresponding micromovements. We hypothesized that when squeezing, we would observe touch traces that move toward the touch points' centroid, and away from it when un-squeezing. If successful, tokens can then be made to behave like a mouse with a button: quickly squeezing and releasing is equivalent to a click; keeping the token squeezed and moving it on the surface is equivalent to a drag. These can be used respectively to trigger discrete events, and to enter quasi-modes. 


\section{Bending Tokens}

Bending a token leads to a state where users are keeping only one finger in contact with the surface (Figure 3-a). As all other token manipulations involve at least two fingers, the number of fingers could be a discriminating factor. However, it is too permissive, as it may also match cases where users lift two fingers off, but leave the token flat on the surface (Figure 3-b). Again, micro-movements may help us detect actual bending actions. We hypothesize that users are likely going to keep their index and middle fingers in contact with the token's side when bending it, while they are going to relax their grip when leaving it flat. We should thus observe still traces before lift-off when bending, as opposed to traces that slightly move away from the centroid in the other case.

\section{COLLECTING TOUCH TRACES}

We collected multi-touch traces of users performing the three types of manipulations described above. Our goal was to gather data about the different finger micro-movements, and to identify criteria that could enable us to recognize the corresponding manipulation events. We were particularly interested in the typical profile of point-to-centroid average distance time-series associated with these movements.

\section{Participants \& Apparatus}

Twelve volunteers (2 female), 23 to 40 year-old (avg. 28.83, med. 28), participated in the data collection. They were seated at a desk, manipulating tokens on a tablet (Samsung SM-T810 Galaxy Tab S2: $237 \times 169$ mm display area / 2048 $\times 1536$ pixels), laid flat on the desk. Participants were videorecorded.

\section{Procedure}

All participants performed the 3 manipulation events: Click and Drag \& Drop, Leave on vs. Lift off and Bend vs. Leave flat. Presentation order was counterbalanced using a Latin Square. All events involved the flexible version of the 6 ToKENS introduced in [9]: 2 circles, 2 squares, 1 triangle, 1 rectangle.

Event $_{1}$ : Click and Drag \& Drop. Participants had to perform 2 types of ACTIONS: Click or Drag. In the Click case, they had to grab the right token using 3 fingers, put it on a black cross, and then slide it toward a red circle located $130 \mathrm{~mm}$ away. Once the token was inside the circle, they had to perform a "click" on the token by compressing it sideways, and then release the pressure. Finally, they removed the token from the surface. In the Drag case, they had to: compress the token right after having put it on the black cross, keep it compressed while moving it toward the red circle, and release the pressure before removing the token from the surface. We collected data involving sliding movements in 4 main DIRECTIONs: up, down, left, right. The tablet was placed in landscape mode for DIRECTION $=\{$ left, right $\}$, and portrait mode for DIRECTION $=\{$ up, down $\}$, so that the red circle would be at the same distance from the black cross in all conditions.

Event $_{2}$ : Leave on vs. Lift off. Participants also had to move a token from a black cross to a red circle. However, once in the circle, participants had to perform one of two ACTIONS:

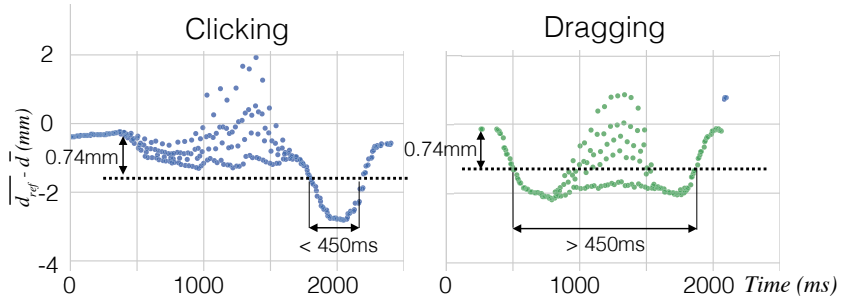

Figure 4. Using Squeeze mode for clicking (left) and dragging (right).

Leave on or Lift off. In the first case, they had to lift their fingers off the surface but leave the token on it. In the second case, they had to lift their fingers, taking the token off the sur-

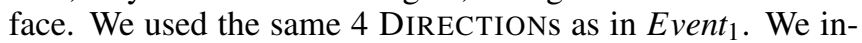
troduced an additional factor, FINGERCOUNT, to capture the two different manipulation styles described in [9]: once a token has been identified with the 3-finger hold, users can keep manipulating it with 3 fingers, or they can relax their grasp and manipulate the token with only 2 fingers. Thus, we had 2 FINGERCOUNT conditions: participants either had to keep their 3 fingers in contact with the surface all along (3-finger condition), or they were asked to lift a finger off the surface after having put the token on the black cross, and to keep it lifted until the end of the trial (2-finger condition). Failure to comply in any given trial meant it had to be performed again.

Event $_{3}$ : Bend vs. Leave flat. The tablet only displayed a black cross. Participants had to put the right token on the surface and perform one of two ACTIONS. In the Bend condition, they had to bend the token, keeping only their thumb in contact with the surface, and then unbend the token by putting the other two fingers back on the surface. In the LeaveFlat condition, they also had to lift two fingers off the tablet, only keeping the thumb in contact, but without bending the token, which remained flat on the tablet. They then had to put their two fingers back on the surface to end the trial.

For each event type, trials are first blocked by ACTION, then by DIRECTION within each ACTION (Event 1 and Event $_{2}$ ), and by FINGERCOUNT within each DIRECTION block $\left(\right.$ Event $\left._{2}\right)$. Each condition is replicated 3 times. Block presentation order is counterbalanced across participants; trial presentation order within a block is random. The whole procedure consists of 252 trials $(72+144+36)$, and lasts approximately one hour.

\section{RECOGNIZERS}

Our main hypothesis was that the micro-movements of interest to us could be observed by looking at the fingers' traces, that should move slightly toward, or away from, the token's center. To verify this, we analyzed, for all collected touch traces, the evolution over time of the average distance $\bar{d}$ of a touch point to the centroid of the corresponding multi-touch sample. In the following, we report the criteria we identified as the most successful for capturing these micro-movements. Parameter values (in bold) are determined in the next section.

1. Squeeze: a token is considered squeezed (Figure 4) when:

$$
\forall i \in\{1 . .|B|\}, \quad \overline{d_{r e f}}-\overline{d_{i}}>\mathbf{d}_{\mathbf{s q z}}
$$


where $\overline{d_{\text {ref }}}$ is the average distance in millimeters of a touch point to the centroid of the corresponding multi-touch sample when users register the token, and $B$ is a buffer containing the successive values of $\bar{d}$ over the last buffer $_{\text {sqz }}$ milliseconds.

2. On/Off: a token is considered as left on the surface when:

$$
m_{\text {end }}>\mathbf{m}_{\text {on_off }}
$$

where $m_{\text {end }}$ is the slope ${ }^{1}$ of the evolution of $\bar{d}$ over the buffer $_{\text {on_off }}$ milliseconds preceding the instant where the last finger has been lifted off the surface (count (fingers) $=0$ ). On the opposite, if $m_{\text {end }} \leq 0$ at this instant, the token is considered as lifted off the surface. Figure 5 illustrates the two cases.

3. Bend: a token is considered as having been bent when:

$$
\max \left(m_{\text {before }},-m_{\text {after }}\right)<0
$$

where $m_{\text {before }}$ (resp. $m_{\text {after }}$ ) is the slope of the evolution of $\bar{d}$ over the buffer $_{\text {bend }}$ milliseconds preceding (resp. following) the instant where only one finger remains in contact with the surface $($ count $($ fingers $)=1$ ) for at least $100 \mathrm{~ms}$, as illustrated in Figure 6. The formula is basically a sign analysis: it checks whether $\bar{d}$ increases or decreases before and after the time span during which there is one single contact point. We initially considered analyzing only $m_{\text {before }}$ to detect when users enter the bent state, but our tests revealed that this sample does not carry enough information to discriminate between bending and leaving flat. This entails that our recognizer considers bent as a discrete event, that gets triggered only once users have unbent the token.

We couple these criteria with state machines that take the number of contact points into account, making it very unlikely that any one event will get confounded with the other two:

- The criterion for squeeze is only evaluated when there are 3 contact points on the surface for at least $200 \mathrm{~ms}$. This is mainly to avoid confusion with cases where users bend the token, as they tend to compress it when unbending.

- The criterion for on/off is only evaluated when the number of contact points becomes null.

- The criterion for bend is only evaluated after a time span of $100 \mathrm{~ms}$ during which there has been exactly 1 contact point.

\section{RECOGNIZER PARAMETERIZATION}

For each of our three micro-movements, we measure the $a c$ curacy of our recognizer by running it on data collected for this micro-movement only. We then test its robustness to false positives by running it on data collected for the other two.

We use the leave-one-out cross-validation technique to parameterize the recognizers: for each participant, we set the parameters to values that maximize the overall recognition score for the 11 other participants. We then report the average score across all 12 participants (mean, median, standard dev.).

Squeezed mode is recognized in 96.9\% (median: 97.9 / std: 3.0) of all trials collected for Event ${ }_{1}$ (with $\mathbf{d}_{\mathbf{s q z}} \in[0.74,0.75]$

\footnotetext{
${ }^{1}$ Computed using the Theil-Sen estimator [11].
}

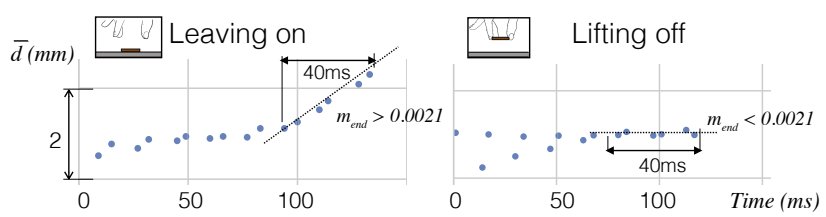

Figure 5. Leaving a token on the surface (left) or lifting it off (right).

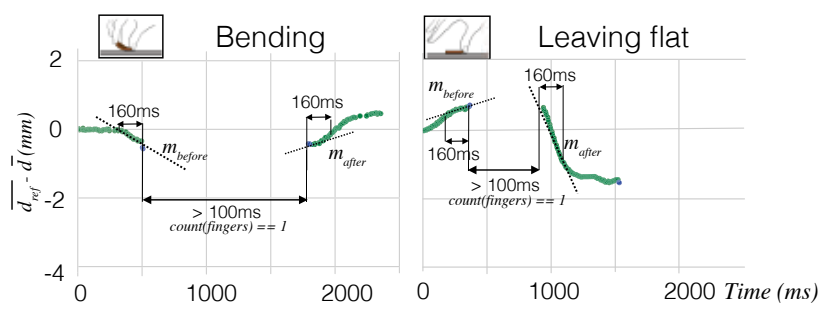

Figure 6. Bending a token (left) or leaving it flat (right).

and buffer $_{\text {sqz }}=100$ ). It is falsely detected in $1.8 \%$ of all trials for Event 2 , and $2.1 \%$ for Event 3 .

States on and off were properly distinguished in $\mathbf{9 0 . 1 \%}$ (median: 92.4 / std: 5.1$)^{2}$ of all trials for Event ${ }_{2}$ (with $\mathbf{m}_{\text {on_off }}$ $\in[0.0018,0.0027]$ and buffer $\left._{\text {on_off }}=40\right)$. The distinction between states on and off also works well for Event 3 , with only $7.6 \%$ of false positives. However, when tested on trials from Event $_{1}$, we observe $43 \%$ of false positives. A finer analysis reveals that the recognizer fails to detect state off right after leaving mode squeezed, which happens when users lift the token off while releasing the pressure applied on the token $(\bar{d}$ increases right before count $($ fingers $)=0)$. Making tokens flexible thus provides opportunities for performing micro-movements in general, but has the side-effect of introducing some ambiguity in this particular case. This is a limitation of our recognizer that we will further investigate. In the meantime, it can be handled by considering the state where count (fingers) $=0$ right after having left mode squeezed as "uncertain", prompting users for input to resolve the ambiguity.

For Event 3 , Bent events were detected in $\mathbf{9 1 . 1 \%}$ (median: 91.7 / std: 6.1) of all trials where ACTION = Bend (with buffer $\left._{\text {bend }} \in[100,160]\right)$. In the remaining $8.9 \%$ trials, the recognizer detected either 0 or at least 2 Bent events (during the same trial). No Bent event is ever accidentally triggered for either Event $t_{1}$ or Event 2 , as the time intervals during which users have only one finger in contact with the surface are infrequent and very short. No Bent event is ever accidentally triggered, either, when ACTION = LeaveFlat .

Finally, some indications about the robustness of our flexibletoken design: we used the same set of six tokens throughout the entire data collection procedure, that consisted of 3024 manipulations by 12 people. No token was broken, or deformed.

\footnotetext{
${ }^{2}$ As a side note, we observed a recognition accuracy close to $90 \%$ for on/off states during informal tests using rigid tokens, suggesting that these micro-movements can also be detected on regular TouchTokens.
} 


\section{CONCLUSION}

As discussed in [9], TouchTokens can play different roles in an application. They can be used to control parameters or filter data in a visualization. They can be used as controllers in games, as data receptacles to hold any kind of content, and even as an access control mechanism. Our new events enable developing more powerful interfaces where tokens can be dragged (squeeze) or clicked (bent, squeezed), and where several tokens can be laid on the surface (on/off enabling the system to keep track of them). This extended vocabulary can be used for different purposes, such as concurrently activating several filters, invoking commands on specific items or transferring data using drag-and-drop, click actions or contextual controls that take the tokens' relative layout into account.

\section{REFERENCES}

1. Patrick Baudisch, Torsten Becker, and Frederik Rudeck. 2010. Lumino: Tangible Blocks for Tabletop Computers Based on Glass Fiber Bundles. In Proceedings of the SIGCHI Conference on Human Factors in Computing Systems (CHI'10). ACM, 1165-1174. DOI : http://dx.doi.org/10.1145/1753326.1753500

2. David Bonnet, Caroline Appert, and Michel Beaudouin-Lafon. 2013. Extending the Vocabulary of Touch Events with ThumbRock. In Proceedings of Graphics Interface 2013 (GI '13). Canadian Information Processing Society, 221-228.

http://dl.acm.org/citation. cfm?id=2532129.2532166

3. Liwei Chan, Stefanie Müller, Anne Roudaut, and Patrick Baudisch. 2012. CapStones and ZebraWidgets: Sensing Stacks of Building Blocks, Dials and Sliders on Capacitive Touch Screens. In Proceedings of the SIGCHI Conference on Human Factors in Computing Systems (CHI'12). ACM, 2189-2192. DOI : http://dx.doi.org/10.1145/2207676.2208371

4. Hans-Christian Jetter, Jens Gerken, Michael Zöllner, Harald Reiterer, and Natasa Milic-Frayling. 2011. Materializing the Query with Facet-streams: A Hybrid Surface for Collaborative Search on Tabletops. In Proceedings of the SIGCHI Conference on Human Factors in Computing Systems (CHI'11). ACM, 3013-3022. DOI : http://dx.doi .org/10.1145/1978942 . 1979390

5. Sergi Jordà, Günter Geiger, Marcos Alonso, and Martin Kaltenbrunner. 2007. The reacTable: Exploring the Synergy Between Live Music Performance and Tabletop Tangible Interfaces. In Proc. TEI '07. ACM, 139-146. DOI : http://dx.doi .org/10.1145/1226969.1226998

6. Jakob Leitner and Michael Haller. 2011. Geckos: Combining Magnets and Pressure Images to Enable New Tangible-object Design and Interaction. In Proceedings of the SIGCHI Conference on Human Factors in Computing Systems (CHI'11). ACM, 2985-2994. DOI : http://dx. doi .org/10.1145/1978942.1979385

7. Rong-Hao Liang, Liwei Chan, Hung-Yu Tseng, Han-Chih Kuo, Da-Yuan Huang, De-Nian Yang, and
Bing-Yu Chen. 2014. GaussBricks: Magnetic Building Blocks for Constructive Tangible Interactions on Portable Displays. In Proceedings of the SIGCHI Conference on Human Factors in Computing Systems (CHI'14). ACM, 3153-3162. DOI : http://dx.doi.org/10.1145/2556288.2557105

8. Rong-Hao Liang, Kai-Yin Cheng, Liwei Chan, Chuan-Xhyuan Peng, Mike Y. Chen, Rung-Huei Liang, De-Nian Yang, and Bing-Yu Chen. 2013. GaussBits: Magnetic Tangible Bits for Portable and Occlusion-free Near-surface Interactions. In CHI'13 Extended Abstracts on Human Factors in Computing Systems (CHI EA '13). ACM, 2837-2838. DOI : http://dx.doi.org/10.1145/2468356.2479537

9. Rafael Morales González, Caroline Appert, Gilles Bailly, and Emmanuel Pietriga. 2016. TouchTokens: Guiding Touch Patterns with Passive Tokens. In Proceedings of the 2016 CHI Conference on Human Factors in Computing Systems (CHI'16). ACM, 4189-4202. DOI :

http://dx.doi.org/10.1145/2858036.2858041

10. Anne Roudaut, Eric Lecolinet, and Yves Guiard. 2009. MicroRolls: Expanding Touch-screen Input Vocabulary by Distinguishing Rolls vs. Slides of the Thumb. In Proceedings of the SIGCHI Conference on Human Factors in Computing Systems (CHI '09). ACM, 927-936. DOI :

http://dx.doi.org/10.1145/1518701.1518843

11. Pranab Kumar Sen. 1968. Estimates of the Regression Coefficient Based on Kendall's Tau. J. Amer. Statist. Assoc. 63, 324 (1968), 1379-1389. DOI: http://dx.doi.org/10.1080/01621459.1968.10480934

12. Simon Voelker, Christian Cherek, Jan Thar, Thorsten Karrer, Christian Thoresen, Kjell Ivar Øvergård, and Jan Borchers. 2015. PERCs: Persistently Trackable Tangibles on Capacitive Multi-Touch Displays. In Proc. UIST' 15. ACM, 351-356. DOI : http://dx.doi.org/10.1145/2807442.2807466

13. Simon Voelker, Kosuke Nakajima, Christian Thoresen, Yuichi Itoh, Kjell Ivar Øvergård, and Jan Borchers. 2013. PUCs: Detecting Transparent, Passive Untouched Capacitive Widgets on Unmodified Multi-touch Displays. In Proc. ITS '13. ACM, 101-104. D0I : http://dx.doi.org/10.1145/2512349.2512791

14. Cary Williams, Xing Dong Yang, Grant Partridge, Joshua Millar-Usiskin, Arkady Major, and Pourang Irani. 2011. TZee: Exploiting the Lighting Properties of Multi-touch Tabletops for Tangible 3D Interactions. In Proceedings of the SIGCHI Conference on Human Factors in Computing Systems (CHI'11). ACM, 1363-1372. DOI : http://dx.doi.org/10.1145/1978942.1979143 